\title{
A systematic review of Analytical Management Techniques applied to Competition Analysis Modeling towards a framework for integrating them with BPM.
}

\author{
Dimitrios A. Karras ${ }^{1}$ and Rallis C. Papademetriou ${ }^{1}$ \\ ${ }^{1}$ Sterea Hellas Institute of Technology, Greece, Automation Dept., \\ Psachna, Evoia, Hellas (Greece) P.C.34400,dakarras@teiste.gr \\ ${ }^{2}$ University of Portsmouth, UK, School of Engineering, Faculty of \\ Technology, Anglesea Building, Anglesea Road, Portsmouth (UK) PO1 3DJ
}

\begin{abstract}
The understanding of Business Process modelling is an essential approach for an Organization or Enterprise to achieve set objectives and improve its operations. Recent development has shown the importance of representing processes to carry out continuous improvement. One important aspect of enterprise modelling is actually its involvement in competition. The modelling and simulation of Business Processes has been able to show Business Analysts, and Managers where bottleneck exists in the system, how to optimize the Business Process to reduce cost of running the Organization, and the required resources needed for an Organization. Although large scale organizations have already been involved in such BPM applications, on the other hand, Small Medium Enterprises (SME) have not drawn much attention with this respect. It seems that SME need more practical tools for modelling and analysis with minimum expenses if possible. One approach to make BPM more applicable to SME but, also, to larger scale organizations would be to properly integrate it with analytical management computational techniques, including the game-theoretic analysis, the Markov-chain modelling and the Cognitive Maps methodology. In BPM research the Petri Nets methodology has already been involved in theory, applications and BPM Software tools. However, this is not the case in the previously mentioned as well as to other analytical management techniques. It is, therefore, important in BPM research to take into account such techniques but focusing on specific modelling requirements. One such requirement is the modelling of market share competition. This paper presents an overview of some important analytical management computational techniques, as the above, that could be integrated in the BPM framework, based on the market share competition analysis paradigm. It provides an overview along with examples of market share competition analysis of the applicability of such methods in the BPM field. The major goal of this systematic overview is to propose steps for the integration of such analytical techniques in the BPM framework so that they could be widely applied.
\end{abstract}

Keywords: Business Process Modelling, Competition analysis of markets, modelling requirements, Analytical Management Techniques, Game-Theory Modelling, Markov-chain modelling, , Cognitive Maps Modelling 


\section{Introduction}

Small and medium-sized enterprises (SMEs) account for more than 90 per cent of the world's enterprises and 50-60 per cent of employment. Their contribution to national and regional economic development and gross domestic product growth is well-recognized (Morsing and Perrini, 2009). In fact, SMEs are often characterized as fostering enhanced local productive capacities; innovation and entrepreneurship; and increased foreign direct investment in both developed and developing countries (Raynard and Forstater, 2002).

Hence, while SMEs account for more than 60 per cent of employment in developing countries, and although they are sometimes portrayed as key vehicles in the struggle against poverty (Luetkenhorst, 2004), there is still a critical lack of knowledge about the extent to which these firms may contribute to the achievement of broader objectives of sustainable and equitable development (Fox, 2005; Jeppesen et al., 2012).

In order to understand the possibility of such a contribution it is important to investigate how SMEs are involving analytical management techniques to better explore their possibilities and systematically optimize their performance in a complex financial world and global market. The focus and interest on complex data management, including big data analytics, has been increased over the recent years in the world of SME firms.

Several research reports attempt, through questionnaires, to understand the use of analytical management and planning tools and techniques in SMEs operating in different countries.

As a result of these studies, the most common used tools and techniques are strategic planning, human resources analysis, total quality management, customer relationship management, outsourcing, financial analysis for firm owners, vision/mission, PEST, financial analysis for competitors, benchmarking, STEP analysis, Porter's 5 forces analysis and analysis of critical success factors. According to Gunn and Williams (2007), the results of their research in the UK, SWOT analysis is the most widely applied strategic tool by all organizations surveyed. Benchmarking was ranked second in terms of its usage by all but manufacturing organizations.

However, it is important to perform a meta-analysis research on all these and most recent reports on the use of management tools and techniques in SMEs in order to clearly answer, in detailed tables, in what extend each technique is involved by SMEs depending on its sector of economy, on its country/continent as well as on other crucial meta-analysis factors.

Moreover, it is frequently noticed that the value of just data has significantly reduced in recent past. There are 2 main factors and open issues to consider:

a) There is an overdose of data and it's really hard for a resource strapped SME to be able to digest it

b) There is an overdose of technology solutions and again it's really hard for SME's to understand this landscape and pick the right solution 
Actionable Insights from data is what everyone, including SMEs, want, something with which, on a daily basis, they can uncover new opportunities to grow their business within a complex world, understanding completely their true performance.

The above two questions have not been answered so far by the research reports for SMEs. These questions are, also, highly correlated to the issue of "on what extend the different analytical management tools are really used by SMEs in the optimization of their performance".

In order, however, for an SME or a larger scale organization to apply such analytical techniques and for the research community to answer the above questions, modelling of the business processes (BPM) involved is absolutely necessary in order to establish a common language, a well-defined framework for the application of analytical management techniques. Therefore, more critical than the meta-analysis previously discussed on the use of data by SMEs and other larger scale organizations, is to review, discuss and provide a framework for the proper integration of BPM methodologies and analytical management techniques worthwhile to be utilized in SMEs and beyond.

The major goal of the paper is, therefore, to discuss suitable analytical management techniques that could be integrated in the BPM framework, and through examples to discuss the feasibility of establishing a well-defined framework for the application of these techniques to enterprises. It is an extension of the paper originally presented in (Karras, Papademetriou, 2017)

With this respect we herein discuss and give examples of game theoretic analysis, probabilistic/stochastic methodology, Markov-chain analysis as well as Cognitive maps methodology in business modelling and analysis towards discussing the feasibility of a well-defined framework for the application of these techniques to SME and larger scale enterprises through the BPM approach.

\section{An Overview of Suitable Analytical Management Techniques for Competition Analysis that could be Integrated in the BPM Methodology}

Most attempts to describe and classify business models in the academic and practice literatures have been taxonomic, that is, developed by abstracting from observations typically of a single industry. With only a few exceptions, these attempts rarely deal fully and properly with all its dimensions of customers, internal organization and monetization; see, for instance, Rappa (2004) and Wirtz et al. (2010). So far, the literature lacks clear typological classifications that are robust to changing context and time (Hempel, 1965). A typology has been proposed that considers four elements Baden-Fuller C. et al. (2010-2013): Identifying the customers (the number of separate customer groups); customer engagement (or the customer proposition); monetization; and value chain and linkages (governance typically concerning the firm internally).

In order to define a framework for the application of analytical management techniques through BPM methodology such a typology of business processes models is important in order to establish the ontologies, the conceptual links as well as the application paradigms. The herein systematic review attempts to describe the aforementioned techniques within this context. 


\subsection{A Markov Chain Business Competition Modelling Analysis}

Many real-world systems, including enterprises functionality and operations, contain uncertainty and evolve over time. Stochastic processes (and Markov chains) are probability models for such systems.

A discrete-time stochastic process is a sequence of random variables $X_{0}, X_{1}, X_{2}, \ldots$ typically denoted by $\left\{X_{n}\right\}$.

The state space of a stochastic process is the set of all values that the $X_{n}$ 's can take. (we will be concerned with stochastic processes with a finite \# of states).

Time: $n=0,1,2, \ldots$.

State: $v$-dimensional vector, $\mathbf{s}=\left(s_{1}, s_{2}, \ldots, s_{v}\right)$

In general, there are $m$ states, $\mathbf{s}^{1}, \mathbf{s}^{2}, \ldots, \mathbf{s}^{m}$ or $\mathbf{s}^{0}, \mathbf{s}^{1}, \ldots, \mathbf{s}^{m-1}$. Also, $X_{n}$ takes one of $m$ values, so $X_{n} \leftrightarrow \mathbf{s}$.

A stochastic process $\left\{X_{n}\right\}$ is called a Markov chain if

$\operatorname{Pr}\left\{X_{n+1}=j \mid X_{0}=k_{0}, \ldots, X_{n-1}=k_{n-1}, X_{n}=i\right\}$

$=\operatorname{Pr}\left\{X_{n+1}=j \mid X_{n}=i\right\} \leftarrow$ transition probabilities

for every $i, j, k_{0}, \ldots, k_{n-1}$ and for every $n$.

Discrete time means $n \in N=\{0,1,2, \ldots\}$.

The future behavior of the system depends only on the current state $i$ and not on any of the previous states. $\operatorname{Pr}\left\{X_{n+1}=j \mid X_{n}=i\right\}=\operatorname{Pr}\left\{X_{1}=j \mid X_{0}=i\right\}$ for all $n$ (They don't change over time)

Normally, stationary Markov chains are considered.

The one-step transition matrix for a Markov chain

with states $\boldsymbol{S}=\{0,1,2\}$ is

where $p_{i j}=\operatorname{Pr}\left\{X_{1}=j \mid X_{0}=i\right\}$

$$
\mathbf{P}=\left[\begin{array}{lll}
p_{00} & p_{01} & p_{02} \\
p_{10} & p_{11} & p_{12} \\
p_{20} & p_{21} & p_{22}
\end{array}\right]
$$

If the state space $S=\{0,1, \ldots, m-1\}$ then we have $\sum_{j} p_{i j}=1 \quad \forall i$ and $p_{i j} \geq 0 \forall i, j$

Potential Studies of Business Modelling based on Markov Chain methodology

- Predict market shares at specific future points in time when different business strategies are applied.

- Assess rates of change in market shares over time.

- Predict market share equilibriums.

- Evaluate the process for introducing new products

In short business competition analysis for market sharing prediction could be benefited from Markov Chain approach.

A relevant example for the application of Markov Chain modeling in the field of SMEs or larger scale organizations, regarding the number of products and thus, the relevant market share switching from enterprise to enterprise, is as follows (adapted from 
https://www.analyticsvidhya.com/blog/2014/07/markov-chain-simplified/):

Let's analyze the competition of three brands, producing the same product (devices, e.g smartphones), examining the number of these products (e.g. the smartphones) switching from brand $i$ in week, for instance, 17 to brand $j$ in week 18

\begin{tabular}{|c|c|c|c|c|c|}
\hline $\begin{array}{l}\text { Smartphone } \\
\text { Producer } \\
\text { Brand }\end{array}$ & $(j)$ & 1 & 2 & 3 & Total \\
\hline (i) & & & & & \\
\hline 1 & & 90 & 7 & 3 & 100 \\
\hline 2 & & 5 & 205 & 40 & 250 \\
\hline 3 & & 30 & 18 & 102 & 150 \\
\hline Total & & 125 & 230 & 145 & 500 \\
\hline
\end{tabular}

This is called the contingency table of the Markov Chain and is used to construct the transition probabilities.

Calculation of the Empirical Transition Probabilities for the smartphone Brand Switching example, $\mathrm{P}=\left(p_{i j}\right)_{I, j=1,2,3}$

\begin{tabular}{|l|cll|}
\hline $\begin{array}{l}\text { Smartphone } \\
\text { Producer } \\
\text { Brand }\end{array}$ & $(j) \quad 1$ & 2 & 3 \\
\hline$(i)$ & & & \\
1 & $90 / 100=0.9$ & $7 / 100=0.07$ & $3 / 100=0.03$ \\
2 & $5 / 250=0.02$ & $205 / 250=0.82$ & $40 / 250=0.16$ \\
3 & & & \\
& $30 / 150=0.2$ & $18 / 150=0.12$ & $102 / 150=0.68$ \\
\hline
\end{tabular}

State variable, $X_{n}=$ brand device purchased in week $n$

$\left\{X_{n}\right\}$ represents a discrete state and discrete time stochastic process, where $S=\{1,2$, $3\}$ and $N=\{0,1,2, \ldots\}$. 
If $\left\{X_{n}\right\}$ has Markovian property and $\mathbf{P}$ is stationary, then a Markov chain should be a reasonable representation of aggregate consumer brand switching behavior

We approximate $\mathrm{q}_{\mathrm{i}}(0)$ by dividing total customers using brand i in week 18 by total sample size of 500, which is let's say the total market at that week.:

$$
\mathbf{q}(0)=\left(\mathrm{q}_{\mathrm{i}}(0), \text { for } \mathrm{i}=1,2,3\right)=(125 / 500,230 / 500,145 / 500)=(0.25,0.46,0.29)
$$

To predict market shares for, say, week 20 (that is, 2 weeks into the future), we simply apply equation with $n=2$ :

$$
\mathbf{q}(2)=\mathbf{q}(0) \mathbf{P}^{(2)}
$$

$$
\mathbf{q}(2)=(0.25,0.46,0.29)\left[\begin{array}{lll}
0.90 & 0.07 & 0.03 \\
0.02 & 0.82 & 0.16 \\
0.20 & 0.12 & 0.68
\end{array}\right]^{2}
$$

$=(0.327,0.406,0.267)$

$=$ expected market share from brands 1,2, 3 at week 20

Property 1: Let $\left\{X_{n}: n=0,1, \ldots\right\}$ be a Markov chain with state space $S$ and statetransition matrix $\mathbf{P}$. Then for $i$ and $j \in S$, and $n=1,2, \ldots$

$$
\operatorname{Pr}\left\{X_{n}=j \mid X_{0}=i\right\}=p_{i j}
$$

where the right-hand side represents the $i j^{\text {th }}$ element of the matrix $\mathbf{P}^{(n)}$.

Property 2: Let $\pi=\left(\pi_{1}, \pi_{2}, \ldots, \pi_{m}\right)$ is the $m$-dimensional row vector of steady-state (unconditional) probabilities for the state space $S=\{1, \ldots, m\}$. To find steady-state probabilities, solve linear system:

$$
\boldsymbol{\pi}=\boldsymbol{\pi} \mathbf{P}, \mathrm{S}_{j=1, m} \pi_{j}=1, \pi_{j} \geq 0, j=1, \ldots, m
$$

$$
\left(\pi_{1}, \pi_{2}, \pi_{3}\right)=\left(\pi_{1}, \pi_{2}, \pi_{3}\right)\left[\begin{array}{lll}
0.90 & 0.07 & 0.03 \\
0.02 & 0.82 & 0.16 \\
0.20 & 0.12 & 0.68
\end{array}\right] \text {, where }
$$

\section{$\pi_{1}+\pi_{2}+\pi_{2}=1, \pi_{1} \geq 0, \pi_{2} \geq 0, \pi_{3} \geq 0$}

$$
\begin{gathered}
\pi_{1}=0.90 \pi_{1}+0.02 \pi_{2}+0.20 \pi_{3} \\
\pi_{2}=0.07 \pi_{1}+0.82 \pi_{2}+0.12 \pi_{3} \\
\pi_{3}=0.03 \pi_{1}+0.16 \pi_{2}+0.68 \pi_{3} \\
\pi_{1}+\pi_{2}+\pi_{3}=1 \\
\pi_{1} \geq 0, \pi_{2} \geq 0, \pi_{3} \geq 0
\end{gathered}
$$

With simple substitutions we could get:

$\boldsymbol{\pi}_{1}=\mathbf{0 . 4 7 4}, \boldsymbol{\pi}_{2}=\mathbf{0 . 3 2 1}, \boldsymbol{\pi}_{3}=\mathbf{0 . 2 0 5}$, which are the steady state calculations.

If we recall that $q_{1}(0)=\mathbf{0 . 2 5}, q_{2}(0)=0.46, q_{3}(0)=0.29$, we could understand the differences between steady state calculations, that is the market share equilibrium in 
the competition between the different brands, and the market share prediction, when competition has not been stabilized.

1. Steady-state predictions of competition analysis between different brands are never achieved in actuality due to a combination of

(i) errors in estimating $\mathrm{P}$

(ii) changes in P over time

(iii) changes in the nature of dependence relationships among the states.

2. Nevertheless, the use of steady-state values is an important diagnostic tool for the decision maker.

3. Steady-state probabilities might not exist unless the Markov chain is ergodic.

\subsection{The Game Theoretic Modelling Analysis Applied to Business Competition Analysis}

Every game has players (usually two), strategies (usually two, but sometimes more) and payoffs (the payoffs to each player are defined for each possible pair of strategies in a two-person game). There are also rules for each game which will define how much information each player knows about the strategy adopted by the other player, when this information is known, whether only pure strategies or mixed strategies may be adopted, etc. etc. Game theory is used to help us think about the strategic interaction between firms in an imperfectly competitive industry. It is particularly helpful for looking at pricing, advertising and investment strategies, and for looking at the decision to enter an industry (and the strategies that can be adopted to deter a firm from entering an industry - entry deterrence) as well as to formulate the outcomes of different strategies of specific business processes. There is a lot of terminology to when someone is first introduced to game theory.

For instance, games can be co-operative or nonco-operative. A co-operative game is one in which the players can form lasting agreements on how to behave. We focus our attention, however, on nonco-operative games in which such binding agreements are not possible, and players are always tempted to cheat on any temporary agreement if they can gain an advantage by cheating. Such games are well suited in the case for modelling different strategies for specific business processes.

Games can be "pure strategy" games or they can allow for "mixed" strategies. Most of the time we will discuss only pure strategy games (for example: if a firm has two strategies for a business process, which are to charge $\$ 50$ and to charge $\$ 100$, then a pure strategy game allows for only these two possibilities). However, we could consider some examples of mixed strategies (for example: if the firm has the two pricing strategies described above, it would also have the option of charging $\$ 50$ thirty percent of the time and charging $\$ 100$ seventy percent of the time - i.e., a probabilistic move). Games can be single-period games or many-period games (many-period games are also called repeated-play games or multi-period games). A single-period game will only be played once and no one thinks about the future possible replaying of the game in making 
their decisions about the best strategy. However, many of life's strategic decisions (for business firms as well as individuals) require us to think about the payoffs that will occur if a game is played over and over and over again. Results in a one-period game can be overturned once you take repeated effects into account.

Games can be described as simultaneous games or sequential games. In a simultaneous game, the two players know what their possible strategies are, they know the identity of the other player, they know what the payoffs are for both players from any combination of strategies, but each player does not know what move the other player has decided to make. In other words, each player knows the incentives, but not the actual strategy adopted. On the other hand, in a sequential game, one player moves first and the other player moves second. The second player to move already knows what strategy the other player has adopted when the second player is making his/her decision.

What constitutes a dominant strategy? A dominant strategy is one that gives you the best result, no matter what the other person chooses to do. For example, consider the following game, adapted from the above brand market share example (note: in all the games herein discussed the payoff of the business process for the first brand Brand\#1 following this process will always be listed first):

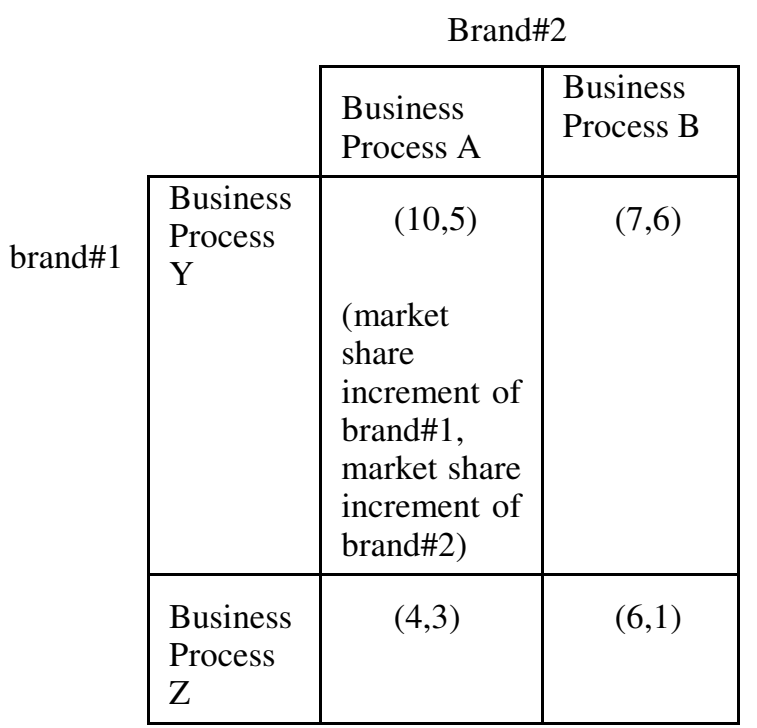

For Brand\#1, $\mathrm{Y}$ is a dominant strategy, because Brand\#1 always ends up with a higher payoff for the enterprise by choosing this business process. For Brand\#2 there is no dominant strategy, because Brand\#2 does better by choosing A if \#1 chooses Z, but Brand\#2 does better by choosing B if \#1 chooses Y.

A Nash equilibrium occurs when neither party has any incentive to change his or her strategy, given the strategy adopted by the

other party. Clearly, the existence of a dominant strategy will result in a Nash equilibrium: in the game above, the enterprise following process 1 always chooses strategy Y; while the enterprise following process 2 then, chooses B; Y,B is a Nash equilibrium. However, games without any dominant strategies also often have Nash equilibria. A game may have no Nash equilibrium, a single Nash equilibrium, or multiple Nash equilibria.

In order for such a methodology to be applied it is important to completely define business processes, payoffs (which, in the competition analysis are the increments/decrements of relevant market shares) and of course the players, which, in our competition analysis case, they are simply the different brands. In our case the 
players are different competitive processes within an enterprise, but they could be within two different firms too. Regarding the payoffs could be even the number of customers attracted by the different strategies. Therefore, the applicability of this analytical management technique should be discussed within BPM framework in order to be established for wide use within SME or larger enterprises.

\subsection{The Cognitive Maps Approach in Modelling Analysis}

Cognitive maps (Axelrod, 1976), (Eden, 1992) are a collection of nodes linked by some arcs or edges. The nodes represent concepts or variables relevant to a given domain. The causal links between these concepts are represented by the edges. The edges are directed to show the direction of influence. Apart from the direction, the other attribute of an edge is its sign, which can be positive (a promoting effect) or negative (an inhibitory effect). Cognitive maps can be pictured as a form of signed directed graph. Figure 1 shows a cognitive map used to represent a scenario involving let's say competition analysis of 7 brands of a specific product $\mathrm{P}$ ( a device, let'say a smartphone), BP1-BP7, following a specific brand switching analysis as the one of section 2.1

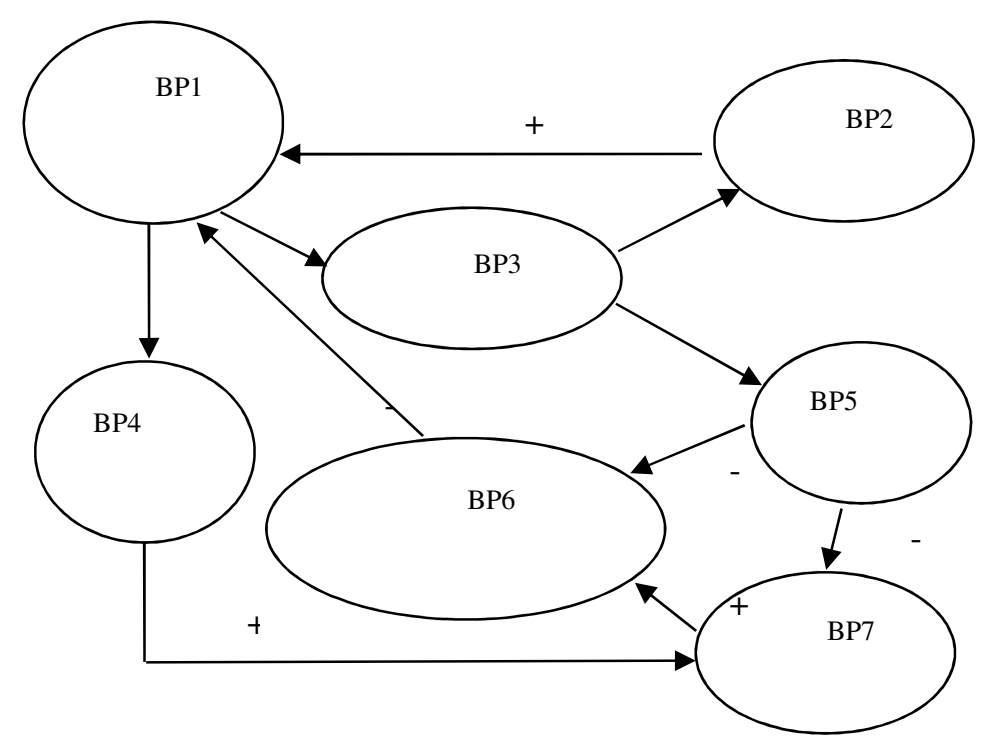

Fig 1. Cognitive map concerning causal relations in a generalization of the Brand Switching example of section 2.1.

The construction of a cognitive map requires the involvement of a knowledge engineer and one or more experts in a given problem domain. Methods for constructing a cognitive map for a relatively recent real-world application are discussed in (Tsadiras, 2003), (Jetter, 2014). 
- Basic structure of FCM

Each node in $\mathrm{C}_{\mathrm{i}}$ in $\mathrm{FCM}$ represents a concept.

Each arc $\left(\mathrm{C}_{\mathrm{i}}, \mathrm{C}_{\mathrm{j}}\right)$ is directed as well as weighted, and represents causal link between concepts, showing how concept $\mathrm{C}_{\mathrm{i}}$ causes concept $\mathrm{C}_{\mathrm{j}}$.

These structures are shown in the following example of a business causal model.

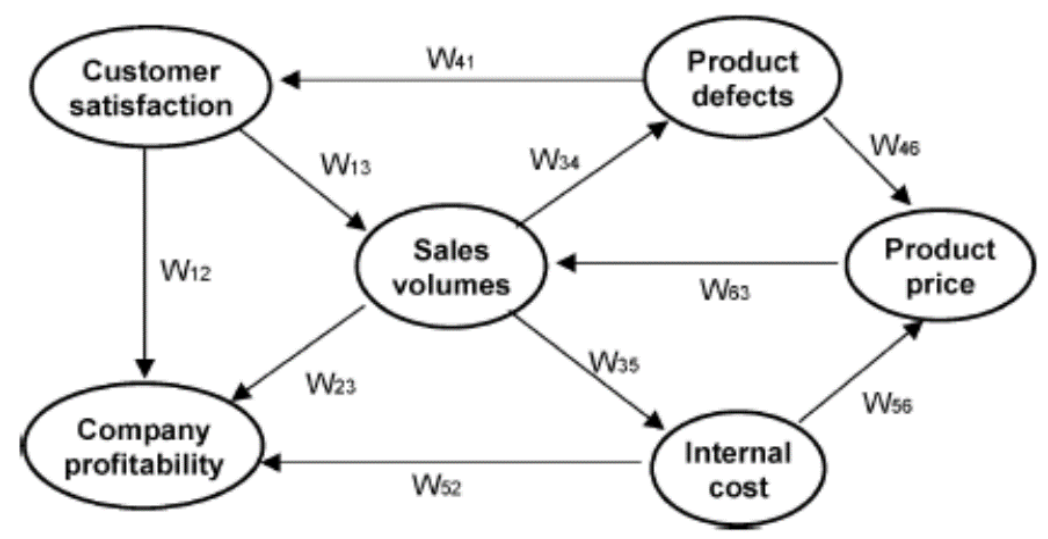

The adjacency matrix related to this causal business model is $\mathrm{W}=$

$\begin{array}{lll}\mathrm{C} 1= & \mathrm{C} 2= & \mathrm{C} 3= \\ \text { Customer } & \text { Product } & \text { Sales } \\ \text { Satisfaction } & \text { defects } & \text { Volumes }\end{array}$
C1
O
O
$\mathrm{VH}$
$\mathrm{C} 2$
VL
O
O
C3
O
H
O
$W=\left\{\begin{array}{l}w_{i j}>0 ; \text { expresses positive causality } \\ w_{i j}=0 ; \text { expresses no causality } \\ w_{i j}<0 ; \text { expresses negative causality }\end{array}\right.$ 


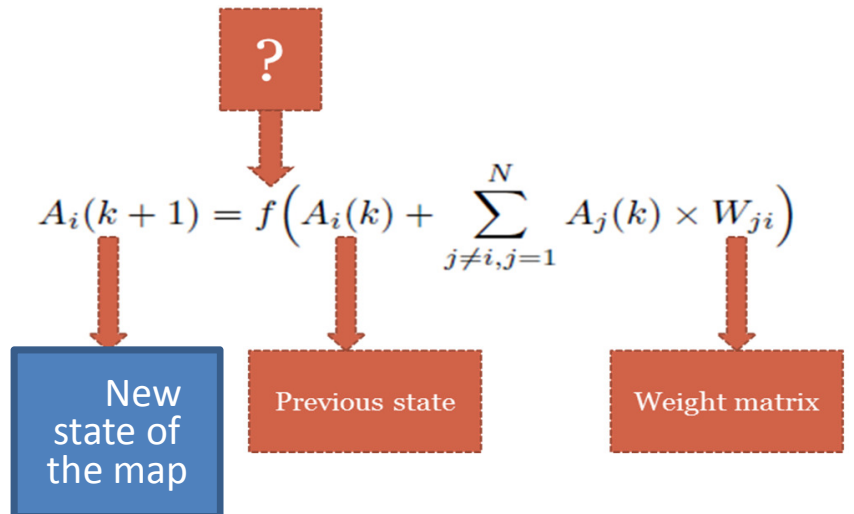

Fig. 2 The evolution of the cognitive map

Fig. 2 shows the evolution of the cognitive map basic equation with the following transfer functions

$$
\begin{aligned}
& f_{\text {sign }}(x)= \begin{cases}1, & x>0, \\
0, & x \leqslant 0 .\end{cases} \\
& f_{\text {tri }}(x)= \begin{cases}1, & x>0, \\
0, & x=0, \\
-1, & x<0 .\end{cases} \\
& f(x)=\tanh (x) \text { or } \\
& f(x)=\frac{\mathrm{e}^{2 x}-1}{\mathrm{e}^{2 x}+1} .
\end{aligned}
$$

FCM Inference Algorithm

Step 1: Definition of the initial vector $\mathbf{A}$ that corresponds to the elements-concepts identified by experts' suggestions and available knowledge.

Step 2: Multiply the initial vector $\mathbf{A}$ with the matrix $\boldsymbol{W}$ defined by experts

Step 3: The resultant vector $\mathbf{A}$ at time step $k$ is updated using function threshold ' $f$ '. Step 4: This new vector is considered as an initial vector in the next iteration.

Step 5: Steps 2-4 are repeated until epsilon (where epsilon is a residual, describing the minimum error difference among the subsequent concepts)

The main objective of building a cognitive map around a problem is to be able to predict the outcome by letting the relevant issues interact with one another. These predictions can be used for finding out whether a decision made by someone is consistent with the whole collection of stated causal assertions. Such use of a cognitive map is based on the assumption that, a person whose belief system is accurately represented in a cognitive map, can be expected to make predictions, decisions and explanations that correspond to those generated from the cognitive map. This leads to the significant 
question: Is it possible to measure a person's beliefs accurately enough to build such a cognitive map? The answer, according to Axelrod and his co-researchers, is a positive one. Formal methods for analysing cognitive maps have been proposed and different methods for deriving cognitive maps have been tried in (Axelrod, 1976).

In a cognitive map, the effect of a node $A$ on another node $B$, linked directly or indirectly to it, is given by the number of negative edges forming the path between the two nodes. The effect is positive if the path has an even number of negative edges, and negative otherwise. It is possible for more than one such paths to exist. If the effects from these paths is a mix of positive and negative influences, the map is said to have an imbalance and the net effect of node $A$ on node $B$ is indeterminate. This calls for the assignment of some sort of weight to each inter-node causal link, and a framework for evaluating combined effects using these numerically weight-ed edges. Fuzzy cognitive maps (FCM) (Caudill, 1990), (Brubaker, 1996a), (Brubaker, 1996b) were proposed as an extension of cognitive maps to provide such a framework.

\section{Fuzzy Cognitive Maps}

The term fuzzy cognitive map (FCM) was coined in (Kosko, 1986) to describe a cognitive map model with two significant characteristics:

(1) Causal relationships between nodes are fuzzified. Instead of only using signs to indicate positive or negative causality, a number is associated with the relationship to express the degree of relationship between two concepts.

(2) The system is dynamic involving feedback, where the effect of change in a concept node affects other nodes, which in turn can affect the node initiating the change. The presence of feedback adds a temporal aspect to the operation of the FCM.

The FCM structure can be viewed as a recurrent artificial neural network, where concepts are represented by neurons and causal relationships by weighted links or edges connecting the neurons.

By using Kosko's conventions, the interconnection strength between two nodes $C_{i}$ and $C_{j}$ is $e_{i j}$, with $e_{i j}$, taking on any value in the range -1 to 1 . Values -1 and 1 represent, respectively, full negative and full positive causality, zero denotes no causal effects and all other values correspond to different fuzzy levels of causal effects. In general, an FCM is described by a connection matrix $E$ whose elements are the connection strengths (or weights) $e_{i j}$. The element in the $i^{t h}$ row and $j^{\text {th }}$ column of matrix $E$ represents the connection strength of the link directed out of node $C_{i}$ and into $C_{j}$. If the value of this link takes on discrete values in the set $\{-1,0,1\}$, it is called a simple FCM. The concept values of nodes $C_{l}, C_{2}, \ldots, C_{n}$ (where $n$ is the number of concepts in the problem domain) together represent the state vector $C$.

An FCM state vector at any point in time gives a snapshot of events (concepts) in the scenario being modelled. In the example FCM shown in figure 2, node $\mathrm{C}_{2}$ relates to the $2^{\text {nd }}$ component of the state vector and the state $\left[\begin{array}{lllllll}0 & 1 & 0 & 0 & 0 & 0 & 0\end{array}\right]$ indicates the event "migration into city" has happened. To let the system evolve, the state vector $C$ is passed repeatedly through the FCM connection matrix $E$. This involves multiplying $C$ by $E$, and then transforming the result as follows:

$C(k+1)=T[C(k) \cdot E]$

where $C(k)$ is the state vector of concepts at some discrete time $k, T$ is the thresholding or nonlinear transformation function, and $E$ is the FCM connection matrix. 
With a thresholding transformation function, the FCM reaches either one of two states after a number of passes. It settles down to a fixed pattern of node values - the so-called hidden pattern or fixed-point attractor. Alternatively, it keeps cycling between a number of fixed states - known as the limit cycle. With a continuous transformation function, a third possibility known as the chaotic attractor (Elert, 1999) exists, when instead of stabilising, the FCM continues to produce different state vector values for each cycle.

\section{Extensions of FCMs}

A number of researchers have developed extended versions of the FCM model described above Tsadiras (2003), Jetter et al. (2014) describe the extended FCM, in which concepts are augmented with memory capabilities and decay mechanisms. The new activation level of a node depends not only on the sum of the weighted influences of other nodes but also on the current activation of the node itself. A decay factor in the interval $[0,1]$ causes a fraction of the current activation to be subtracted from itself at each time step.

Park (1995) introduces the FTCM (Fuzzy Time Cognitive Map), which allows a time delay before a node $x_{i}$ has an effect on node $x_{j}$ connected to it through a causal link. The time lags can be expressed in fuzzy relative terms such as "immediate", "normal" and "long" by a domain expert. These terms can be assigned numerical values such as 1, 2, 3 . If the time lag on a causal link $e_{i j}$ is $\mathrm{m}(1 \geq m)$ delay units, then $m-1$ dummy nodes

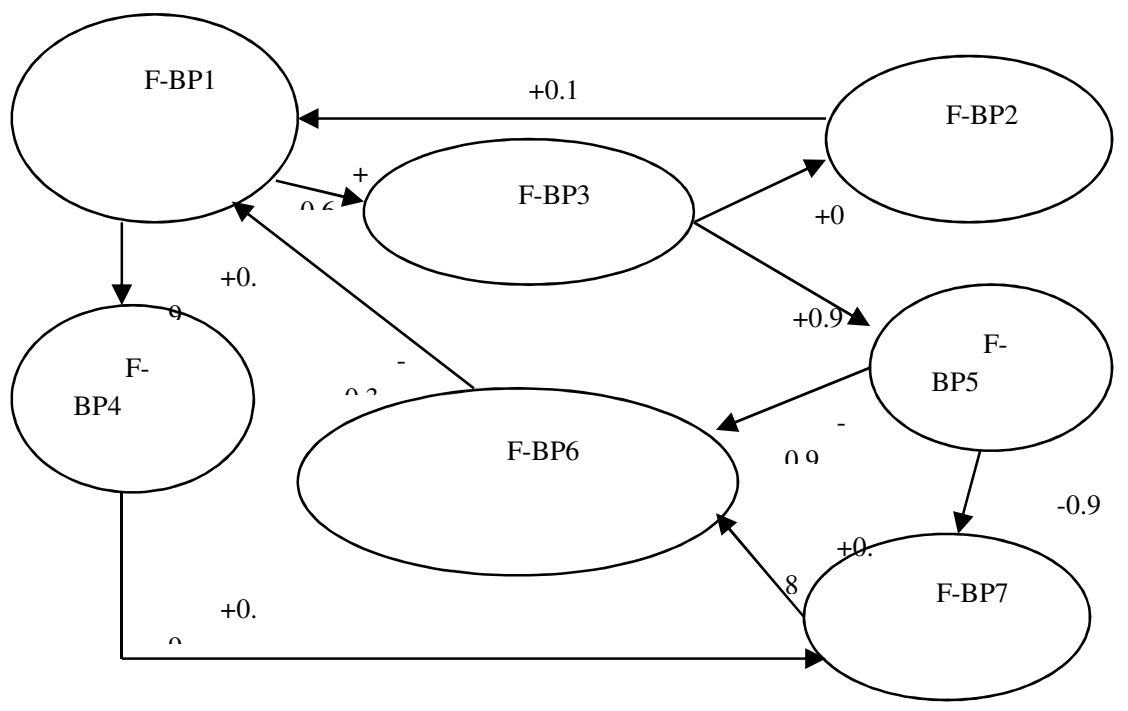

Fig 3. Fuzzified version of the cognitive map shown in figure 1.

are introduced between node $i$ and node $j$.

Decision makers often find it difficult to cope with significant real-world systems. 
These systems are usually characterised by a number of concepts or facts interrelated in complex ways. They are often dynamic ie, they evolve through a series of interactions among related concepts. Feedback plays a prominent role among them by propagating causal influences in complicated pathways. Formulating a quantitative mathematical model for such a system may be difficult or impossible due to lack of numerical data, its unstructured nature, and dependence on imprecise verbal expressions. FCMs provide a formal tool for representing and analysing such systems with the goal of aiding decision making.

Given an FCM's edge matrix and an input stimulus in the form of a state vector, each of the three possible outcomes mentioned above can provide an answer to a causal "what if" question. The inference mechanism of FCMs works as follows. The node activation values representing different concepts in a problem domain are set based on the current state. The FCM nodes are then allowed to interact (implemented through the repeated matrix multiplication mentioned above). This interaction continues until:

(1) The FCM stabilises to a fixed state (the fixed-point attractor), in which some of the concepts are 'on' and others are not.

(2) A limit cycle is reached.

(3) The FCM moves into a chaotic attractor state instead of stabilising as in (1) and (2) above.

The usefulness of the three different types of outcomes depends on the user's objectives. A fixed-point attractor can provide straightforward answers to causal "what if" questions. The equilibrium state can be used to predict the future state of the system being modelled by the FCM for a particular initial state. As an example based on figure

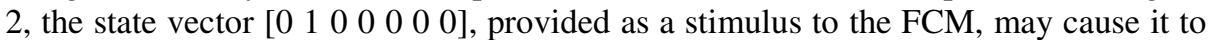
equilibrate to the fixed-point attractor at $\left[\begin{array}{lllllll}0 & 0 & 0 & 1 & 0 & 0 & 0\end{array}\right]$. Such an equilibrium state would indicate that an increase in "migration into city" eventually leads to the increase of "garbage per area".

A limit cycle provides the user with a deterministic behaviour of the real-life situation being modelled. It allows the prediction of a cycle of events that the system will find itself in, given an initial state and a causal link (edge) matrix. For FCMs with continuous transformation function and concept values, a resulting chaotic attractor can assist in simulation by feeding the simulation environment with endless sets of events so that a realistic effect can be obtained.

\section{Development of FCMs for decision modelling}

FCMs can be based on textual descriptions given by an expert on a problem scenario or on interviews with the expert. The steps followed are:

Step 1: Identification of key concepts/issues/factors influencing the problem.

Step 2: Identification of causal relationships among these concepts/issues/factors.

Experts give qualitative estimates of the strengths associated with edges linking nodes. These estimates are translated into numeric values in the range -1 to 1 . For example, if an increase in the value of concept $A$ causes concept $B$ to increase significantly (a strong positive influence), a value of 0.8 may be associated with the causal link leading from 
$A$ to $B$. Experts themselves may be asked to assign these numerical values. The outcome of this exercise is a diagrammatic representation of the FCM, which is converted into the corresponding edge matrix.

\section{Learning in FCMs}

FCM learning involves updating the strengths of causal links. Combining multiple FCMs is the simplest form of learning. An alternative learning strategy is to improve the FCM by fine-tuning its initial causal link or edge strengths through training similar to that in artificial neural networks. Both these approaches are outlined below.

Multiple FCMs constructed by different experts can be combined to form a new FCM. FCM combination can provide the following advantages:

1. It allows the expansion of an FCM by incorporating new knowledge embodied in other FCMs.

2. It facilitates the construction of a relatively bias-free FCM by merging different FCMs representing belief systems of a number of experts in the same problem domain.

The procedures for combining FCM are outlined in (Kosko, 1988). Generally, combination of FCMs involves summing the matrices that represent the different FCMs. The matrices are augmented to ensure conformity in addition. Each FCM drawn by different experts may be assigned a credibility weight. The combined FCM is given by

$$
\mathrm{E}=\sum_{k=1}^{k=N} \mathrm{~W}_{k} \mathrm{E}_{k}
$$

Where $\mathrm{E}$ is the edge matrix of the new combined FCM, $\mathrm{E}_{k}$ is the edge matrix of FCM $k, \mathrm{~W}_{k}$ is the credibility weight assigned to FCM $k$, and $N$ is the number of FCMs to be combined. Siegel and Taber (1987) outlines procedures for credibility weights assignment in FCMs.

McNeill and Thro (1994) discuss the training of FCMs for prediction. A list of state vectors is supplied as historical data. An initial FCM is constructed with arbitrary weight values. It is then trained to make predictions of future average value in a stock market using historical stock data. The FCM runs through the historical data set one state at a time. For each input state, the 'error' is determined by comparing the FCM's output with the expected output provided in the historical data. Weights are adjusted when error is identified. The data set is cycled until the error has been reduced sufficiently for no more changes in weights to occur.

If a correlated change between two concepts is observed, then a causal relation between the two is likely and the strength of this relationship should depend on the rate of the 
correlated change. This proposition forms the basis of the Differential Hebbian Learning (DHL). Kosko (1992) discusses the use of DHL as a form of unsupervised learning for FCMs. DHL can simplify the construction of FCMs by allowing the expert to enter approximate values (or even just the signs) for causal link strengths. DHL can then be used to encode some training data to improve the FCM's representation of the problem domain and consequently its performance.

\section{Business Models as Cognitive Maps}

Drawing on the insights of the cognitive mapping approach in strategic management, we argue that the causal structures embedded in business models can be usefully conceptualized and represented as cognitive maps (Furnari S., 2015). From this perspective, a business model's cognitive map is a graphical representation of an entrepreneur or top manager's beliefs about the causal relationships inherent in that business model (Furnari S., 2015). By emphasizing the causal nature of business models, this definition is consistent with previous studies viewing business models as sets of choices and the consequences of those choices (e.g. Casadesus-Masanell \& Ricart, 2010), and with studies that explicitly highlight the importance of cause-effect relationships in business models' cognitive representations (e.g. Baden-Fuller \& Haefliger, 2013; Baden-Fuller \& Mangematin, 2013). Business models' cognitive maps can be derived from the texts that entrepreneurs and top managers use in designing their business models, or to pitch their projects to various audiences (including investors, customers, policy makers); or they can be derived from primary interviews with entrepreneurs and top managers (Furnari S., 2015). Thus, the content of a business model's cognitive map can be idiosyncratic, depending on the particular individual's cognitive schemas and on the language they use. The raw concepts that entrepreneurs and top managers use in their causal statements identify the elements of a business model's cognitive map that are induced empirically (Furnari S., 2015). At the same time, such maps may include elements deduced theoretically from extant theories about business models - i.e. the conceptual categories developed in such theories (such as "value proposition", "monetization mechanisms") - that can be useful to classify the raw concepts used by entrepreneurs and top managers, providing a basis for comparing different individuals' cognitive maps Thus, business models' cognitive maps include both inductive and deductive elements, as do other types of cognitive maps (e.g. Axelrod, 1976; Bryson et al., 2004).

For the sake of illustrating examples of business models' cognitive maps, we focus particularly on the business model representation developed by Baden-Fuller and Mangematin (2013), (Furnari S., 2015). Among the several business model representations suggested in the literature, we adopt this typological representation because it strikes a balance between parsimony and generality, thus meeting the criteria typically recommended for solid theory-based typologies (e.g. Doty \& Glick, 1994; Delbridge \& Fiss, 2013). Specifically, this typology includes the essential building blocks of the business model as covered by other business model representations, thus having a general scope in terms of content. At the same time, it uses a more parsimonious set of categories than other business model representations in covering this general scope. For this reason, in the cognitive maps' illustrations provided below, we used the four constructs characterizing this business model representation 
("customer identification", "customer engagement (or value proposition)", "value chain" and "monetization") as organizing categories (Furnari S., 2015). Although we use this specific business model representation here for illustrating business models' cognitive maps, the cognitive mapping approach developed in this paper can be used, more generally, with any other business model representation, depending on the analyst's preferences and research objectives (Furnari S., 2015).

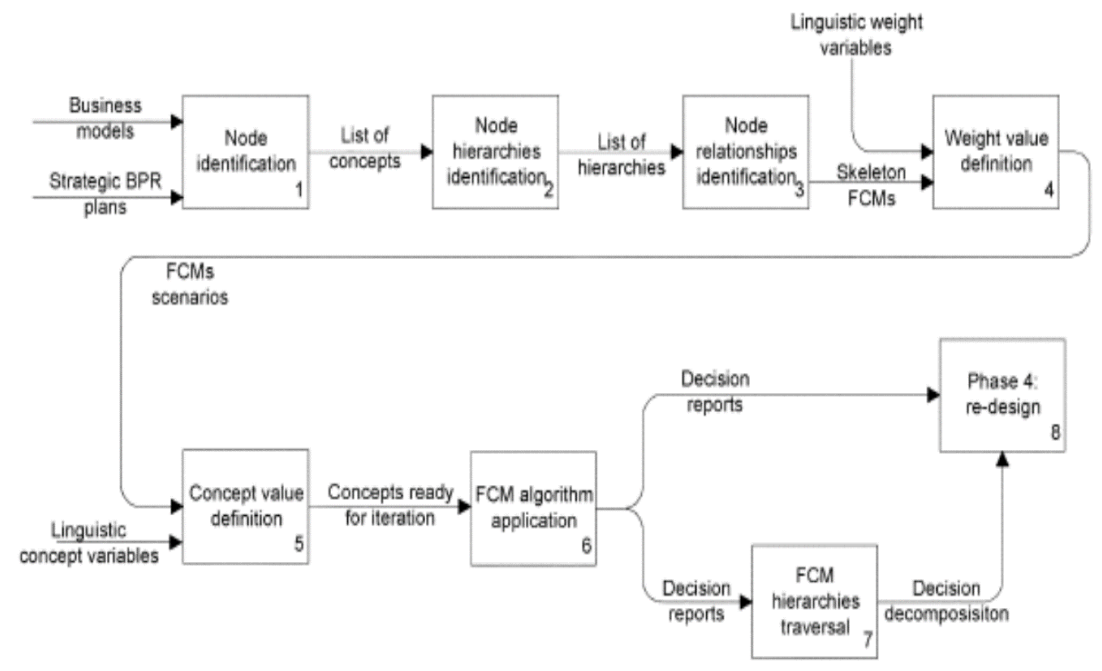

Fig 4 A model for integrating FCM or Cognitive Maps approach in BPM.

\section{Discussion - Conclusions}

In this study we have attempted to present and analyse some important analytical management techniques that might be of value in Business Process Modelling. We have argued through examples relevant to Business Competition analysis of different brands sharing the same market, that each such technique could be involved in business process analysis via specific formalisms and that in order for these techniques to be widely utilized by enterprises a common well defined framework should be established based on BPMN. BPMN could provide the representation schemes that should be integrated in the associated formalisms, as shown in the next diagram.

The Basic elements of BPMN (including BPMN 2.0)—A Proposal towards a framework for integrating analytical management techniques
Activities
Events
Gateways
Connectors

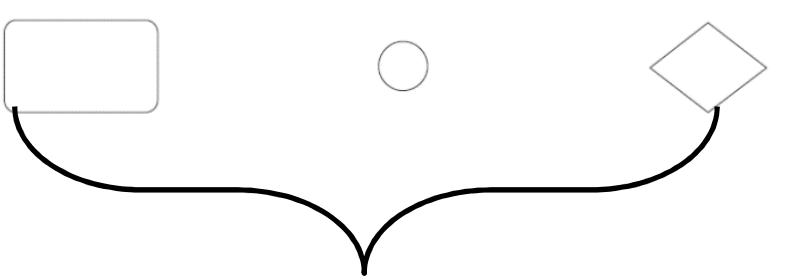




\section{Flow objects BPMN 2.0}

An enriched Activity in BPMN 2.0 is a possible conveyor for integrating analytical management techniques in Business Process Modelling - in combination with GATEWAYS for decisions based on the results of such enriched activities

To the above discussed end, our presentation is a first step. Each analytical management technique herein presented should be analysed in depth in order to be integrated with BPM methodology in a common useful and well organized application framework, that in the sequel could be employed in real world scenarios, managing even big data of the associated enterprises.

\section{REFERENCES}

Ashcroft M. (2012), Bayesian networks in business analytics, [in:] M. Ganzha, L. Maciaszek, M. Paprzycki (Eds.), Proceedings of the Federated Conference on Computer Science and Information Systems FedCSIS 2012, Polskie Towarzystwo Informatyczne, IEEE Computer Society Press, Warsaw, Los Alamitos, CA 2012, pp. 955-961.

Anderson RD, Mackoy RD, Thompson VB, Harrell G. (2004), A Bayesian network estimation of the service-profit chain for transport service satisfaction. Decis Sci. 2004;35(4):665-89.

Axelrod R.M. (Ed.).(1976). Structure of decision: The cognitive maps of political elites. Princeton: Princeton University press.

Baden-Fuller C. \& Morgan M. (2010). Business Models as Models, Long Range Planning, 43(23): $156-171$

Baden-Fuller C. \& Haefliger S. (2013). Business models and technological innovation, Long Range Planning, 46(6): 419-426.

Baden-Fuller C., \& Mangematin V. (2013). Business models: A challenging agenda, Strategic Organization, 11(4), 418-427.

Barr P.S., Stimpert J.L. \& Huff A.S. (1992). Cognitive change, strategic action, and organizational renewal. Strategic management journal, 13(S1): 15-36.

Bhargava, H. K., Herrick, C., Sridhar, S. (1999) "Beyond Spreadsheets: Tools for Building Decision Support Systems", IEEE Expert, 32(3), 1999.

Brubaker, D. (1996a) "Fuzzy Cognitive Maps", EDN Access, April 11 1996, [WWW document]. URL http://www.ednmag.com

Brubaker, D. (1996b) "More on Fuzzy Cognitive Maps", EDN Access, April 25 1996b, [WWW document]. URL http://www.ednmag.com

Bryson J.M., Ackermann F., Eden C. \& Finn, C.B. (2004). Visible thinking: Unlocking causal mapping for practical business results, John Wiley \& Sons.

Calori R., Johnson G. \& Sarnin P. (1994). CEOs' cognitive maps and the scope of the organization, Strategic Management Journal, 15: 437-457.

Carley K. \& Palmquist M. (1992). Extracting, representing, and analyzing mental models. Social forces, 70(3): 601-636.

Casadesus-Masanell R. \& Ricart J.E. (2010). From Strategy to Business Models and onto Tactics. Long Range Planning, 43(2-3): 195-215.

Caudill, M. (1990) "Using Neural Nets: Fuzzy Cognitive Maps”, AI Expert, June 1990, pp. 4953. 
Chakraborty S., et. al. (2016), A Bayesian Network-based customer satisfaction model: a tool for management decisions in railway transport, Decision Analytics, 2016, 3:4,DOI: 10.1186/s40165-016-0021-2, published:9/2016

Chesbrough H. \& Rosenbloom R.S. (2002). The role of the business model in capturing value from innovation: Evidence from Xerox Corporation's technology spin-off companies. Industrial and Corporate Change, 11(3): 529-555.

Chong, A., Khan, S., Gedeon, T. (1999a) "Differential Hebbian Learning in Fuzzy Cognitive Maps: A Methodological View in the Decision Support Perspective", Proc. Third AustraliaJapan Joint Workshop on Intelligent and Evolutionary Systems, 23-25 Nov. 1999, Canberra (to be published).

Chong, A. (1999b) "Development of a Fuzzy Cognitive Map Based Decision Support Systems Generator", Honours dissertation, School of Information Technology, Murdoch University, 1999.

Clarke, I., \& Mackaness, W. (2001). Management 'intuition': an interpretative account of structure and content of decision schemas using cognitive maps. Journal of Management Studies, 38(2): 147-172.

Daly R, Shen Q, Aitken S. (2011), Learning Bayesian networks: approaches and issues. Knowl Eng Rev. 2011;26(02):99-157.

Davis G.F. \& Marquis C. (2005). Prospects for organization theory in the early twenty-first century: Institutional fields and mechanisms. Organization Science, 16(4): 332-343.

Delbridge R. \& Fiss P.C. (2013). Editors' comments: styles of theorizing and the social organization of knowledge. Academy of Management Review, 38(3): 325-331.

Doganova L. \& Eyquem-Renault M. (2009). What do business models do?: Innovation devices in technology entrepreneurship. Research Policy, 38(10): 1559-1570.

Doty D.H. \& Glick W.H. (1994). Typologies as a unique form of theory building: Toward improved understanding and modeling. Academy of management review, 19(2): 230-251.

Doz Y.L. \& Kosonen M. (2010). Embedding Strategic Agility: A Leadership Agenda for Accelerating Business Model Renewal. Long Range Planning, 43(2-3): 370-382.

Eden C., Ackermann F. \& Cropper S. (1992). The analysis of cause maps. Journal of Management Studies, 29(3): 309-324.

Eisenmann T., Parker G. \& Van Alstyne M.W. (2006). Strategies for two-sided markets. Harvard business review, 84(10): 92.

Elert, G. (1999) The Chaos Hypertexbook, 1999, http://hypertextbook.com/chaos/about.shtml

Elster J. (1998). A plea for mechanisms. in Hedström, P., \& Swedberg, R. (Eds.). Social mechanisms: An analytical approach to social theory. Cambridge University Press.

Fiol C.M. \& Huff A.S. (1992). Maps for managers: where are we? Where do we go from here? Journal of Management Studies, 29(3): 267-285.

Fox, T. (2005), Small and Medium-sized Enterprises (SMEs) and Corporate Social Responsibility - A Discussion Paper, International Institute for Environment and Development, London.

Furnari, S. (2015), A Cognitive Mapping Approach to Business Models: Representing Causal Structures and Mechanisms, Chapter 8 in Business Models and Modelling; Volume 33; Advances in Strategic Management editors C. Baden-Fuller and V. Mangematin; Emerald Press, 2015

Gavetti G.M., Henderson R. \& Giorgi S. (2005) Kodak and The Digital Revolution (A). Harvard Business School Case \#705-448.

Goldman I. (2012) 'Poor decisions that humbled the Kodak giant'. Financial Times. 13 April.

Grandori A. \& Furnari S. (2008), A chemistry of organization: Combinatory analysis and design, Organization Studies, 29(2): 315-341. 
Gunn R. and Williams W. (2007), Strategic tools: an empirical investigation into strategy in practice in the UK, Strat. Change 16: 201-216 (2007),

Published

online in Wiley InterScience (www.interscience.wiley.com) DOI: 10.1002/jsc.799

Heckerman D, Geiger D, Chickering DM. (1995), Learning Bayesian networks: the combination of knowledge and statistical data. Mach Learn. 1995;20:197-243

Hempel, C. G. (1965) 'Fundamentals of Taxonomy, and Typological Methods in the Natural and Social Sciences', Aspects of Scientific Explanation, ch. 6., pp. 137-72. New York: Macmillan.

Hodgkinson G.P., Bown N.J., Maule A.J., Glaister K.W. \& Pearman A.D. (1999). Breaking the frame: An analysis of strategic cognition and decision making under uncertainty, Strategic Management Journal, 20: 977-985.

Hodgkinson G.P. \& Healey M.P. (2008). Cognition in organizations, Annual Review of Psychology, 59: 387-417.

Hodgkinson G.P., Maule A.J. \& Bown, N.J. (2004). Causal cognitive mapping in the organizational strategy field: A comparison of alternative elicitation procedures, Organizational Research Methods, 7(1): 3-26.

Huff A.S. (1990). Mapping strategic thought. Chichester, UK: Wiley.

Huff A.S., Narapareddy V. \& Fletcher K.E. (1990). Coding the causal association of concepts in Huff, A.S. Mapping strategic thought, New York: John Wiley and Sons.

Jenkins M. \& Johnson G. (1997). Entrepreneurial intentions and outcomes: A comparative causal mapping study. Journal of Management Studies, 34: 895-920.

Jensen FV, Nielsen TD. (2007), Bayesian networks and decision graphs. 2nd ed. Berlin: Springer; 2007

Jeppesen, S., Kothuis, B. and Tran, A.N. (2012), Corporate Social Responsibility and Competitiveness for SMEs in Developing Countries: South Africa and Vietnam, Focales 16, Agence Francaise de Development, Paris.

Jetter Antonie J. and Kok K. (2014), Fuzzy Cognitive Maps for futures studies-A methodological assessment of concepts and methods, Futures journal, Volume 61, September 2014, Pages 45-57, Elsevier, https://doi.org/10.1016/j.futures.2014.05.002

Johnson S, Fielding F, Hamilton G, Mengersen K. (2010), An integrated Bayesian network approach to lyngbya majuscula bloom initiation. Mar Environ Res. 2010;69(1):27-37.

Kaplan S. \& Sawhney M. (2000). B2B E-Commerce hubs: towards a taxonomy of business models. Harvard Business Review, 79(3): 97-100.

Kardaras, D., and Karakostas, B. (1999) "The use of Fuzzy Cognitive Maps to Simulate Information Systems Strategic Planning Process", Information and Software Technology, Vol.41, Issue 4, 1999, pp. 197-210.

Karras, D.A., Papademetriou, R.C. (2017), “A systematic review of analytical management techniques in business process modelling for smes beyond what-if-analysis and towards a framework for integrating them with BPM “, BMSD 2017 - Proceedings of the 7th International Symposium on Business Modeling and Software Design

Klang D., Wallnöfer M. \& Hacklin F. (2014). The Business Model Paradox: A Systematic Review and Exploration of Antecedents, International Journal of Management Reviews.

Kosko, B. (1986) "Fuzzy Cognitive Maps", Int. J. Man-Machine Studies, Vol.24, 1986, pp.6575.

Kosko, B. (1992) "Neural Networks and Fuzzy Systems", Prentice Hall, Englewood Cliffs, NJ, 1992.

Lee, K.C., Kim, H. S. (1997) "A Fuzzy Cognitive Map-based Bi-directional Inference Mechanism: An Application to Stock Investment Analysis", Intelligent Systems in Accounting, Finance \& Management, 6, 1997, pp. 41-57. 
Lee, K.C., Lee, W.J., Kwon, O.B., Han, J.H., Yu, P.I. (1998) "Strategic Planning Simulation Based on Fuzzy Cognitive Map Knowledge and Differential Game", Simulation, Nov. 1998, pp. 316-327.

Laukkanen M. (1994). Comparative cause mapping of organizational cognitions, Organization science, 5(3): 322-343.

Luetkenhorst, W. (2004), "Corporate social responsibility and the development agenda",Intereconomics, Vol. 39 No. 3, pp. 157-166.

McNeill, M. F., and Thro, E. (1994) "Fuzzy Logic: A Practical Approach", AP Professional Boston 1994.

Morsing, M. \& Perrini, F., 2009. CSR in SMEs: do SMEs matter for the CSR agenda?. Business Ethics: A European Review, 18(1).

Nadkarni S, Shenoy PP. (2001) A Bayesian network approach to making inferences in causal maps. Eur J Oper Res. 2001;128(3):479-98.

Nadkarni S, Shenoy PP. (2004) A causal mapping approach to constructing Bayesian networks. Dec Support Syst. 2004;38(2):259-81.

Park, K.S. (1995) "Fuzzy Cognitive Maps Considering Time Relationships", International Journal of Human-Computer Studies, Feb 1995, pp. 157-167.

Pearl J. (1988) Probabilistic reasoning in intelligent systems. San Francisco: Morgan Kaufmann Publishers Inc; 1988.

Power, D.J. (1997) "What is DSS?" DS*, The On-Line Executive Journal for Data-Intensive Decision Support, Vol.1, No.3, October 21, 1997, [WWW document]. URL: http://dss.cba.uni.edu/ papers/whatisadss

Rappa, M. A. (2004) 'The Utility Business Model and the Future of Computing Services', IBM Systems Journal 43(1) 32-42.

Raynard, P. and Forstater, M. (2002), "Corporate social responsibility: implications for small and medium enterprises in developing countries", UNIDO, Geneva, available at: www.unido.org/fileadmin/import /29959_CSR.pdf (accessed 27 September 2016).

Render, B., and Stair, M.S. (1988) "Quantitative Analysis for Management", Allyn \& Bacon, Boston 1988.

Salini S, Kenett RS. (2009), Bayesian networks of customer satisfaction survey data. J Appl Stat. 2009;36(11):1177-89

Taber, R. (1991) "Knowledge Processing with Fuzzy Cognitive Maps", Expert Systems With Applications, Vol. 2, 1991, pp. 83-87.

Tsadiras, Athanasios K. "Using fuzzy cognitive maps for e-commerce strategic planning." Proc. 9th Panhellenic Conf. on Informatics (EPY'2003). 2003.

Turban, E. (1993) Decision Support and Expert Systems Management Support Systems, 3rd ed., Macmillan, New York 1993.

VidHya Analytics (2017), Introduction to Markov chain : simplified!, (https://www.analyticsvidhya.com/blog/2014/07/markov-chain-simplified/) accessed 1/2017

Wirtz, B. W., Schilke, O. and Ullrich, S. (2010) 'Strategic Development of Business Models: Implications of the Web 2.0 for Creating Value on the Internet', Long Range Planning 43(23): 272-90. 